\title{
Airway management using laryngeal mask airway in insertion of the Montgomery tracheal tube for subglottic stenosis
} \section{-A case report-}

\author{
Jung Sun Park ${ }^{1}$, Young-Suk Kwon ${ }^{1}$, Sangseock Lee ${ }^{1}$, Jun Heum Yon ${ }^{1}$, and Dong Won Kim² \\ Departments of ${ }^{1}$ Anesthesiology and Pain Medicine, ${ }^{2}$ Thoracic Surgery, Sanggye Paik Hostpital, Inje University School of Medicine, \\ Seoul, Korea
}

The Montgomery tracheal tube (T-tube) is a device used as a combined tracheal stent and airway after laryngotracheoplasty for patients with tracheal stenosis. This device can present various challenges to anesthesiologists during its placement, including the potential for acute loss of the airway, inadequate administration of inhalation agents, and inadequacy of controlled mechanical ventilation. The present case of successful airway management used a laryngeal mask airway under total intravenous anesthesia with propofol and remifentanil in the insertion of a Montgomery T-tube in a tracheal resection and thyrotracheal anastomosis because of severe subglottic stenosis. (Korean J Anesthesiol 2010; 59: S33-S36)

Key Words: Laryngeal mask airway, Montgomery T-tube, Subglottic stenosis.

During the management of general anesthesia for patients undergoing laryngotracheal surgery, anesthesiologists may have to deal with patients who need insertion of a Montgomery tube or with cases that have such a tube in situ. The Montgomery tracheal tube (T-tube) is a device used as a combination tracheal stent and tracheostomy tube to prevent postoperative tracheal stenosis [1]. This device, which was introduced in 1960 to support the trachea during laryngotracheoplasty, is an uncuffed silicone T-tube that is inserted with a long limb into the trachea and with a short limb protruding through the tracheostomy stoma. Anesthesiologists who are unfamiliar with this device may experience problems with air dilution, hypoventilation, and patient awareness during anesthesia [2]. Only a few reports to date have described the anesthetic management of patients requiring Montgomery tube insertion [3]. The present reports describe a case of successful airway management using laryngeal mask airway in the insertion of a Montgomery T-tube for a patient who underwent tracheal resection and thyrotracheal anastomosis because of severe subglottic stenosis.

\section{Case Report}

A 71-year-old woman was admitted for acute sub-dural

Received: May 11, 2010. Revised: 1st, June 2, 2010; 2nd, August 7, 2010. Accepted: August 23, 2010.

Corresponding author: Sangseock Lee, M.D., Department of Anesthesiology and Pain Medicine, Sanggye Paik Hospital, Inje University School of Medicine, Sanggye 7-dong, Nowon-gu, Seoul 139-707, Korea. Tel: 82-2-950-1173, Fax: 82-2-950-1323, E-mail: sslee@paik.ac.kr This was presented at the $86^{\text {th }}$ Annual Scientific Meeting of the Korean Society of Anesthesiologists, 2009, International Convention Center, Jeju, Korea. @ This is an open-access article distributed under the terms of the Creative Commons Attribution Non-Commercial License (http:// creativecommons.org/licenses/by-nc/3.0/), which permits unrestricted non-commercial use, distribution, and reproduction in any medium, provided the original work is properly cited. 
hematoma in the left fronto-temporo-parietal cerebral area. The patient had a medical history of cerebella infarction, atrial fibrillation and diabetes mellitus. The patient had previously undergone a craniotomy with hematoma removal and tracheostomy. Seven days after tracheostomy, the patient complained of occasional dyspnea. T-stoma granulation occurred 60 days after the tracheostomy. Seventy days postoperatively, the patient was operated on to remove the granulation. Despite this, the dyspnea worsened and oxygen saturation $\left(\mathrm{SpO}_{2}\right)$ occasionally dropped to $75 \%$. Subglottic stenosis was confirmed, which required tracheal resection and end-to-end anastomosis 4 months after the tracheostomy. However, the length of the stenotic lesion was too long and its position was too high, prompting the surgeon to attempt a tracheal resection and thyrotracheal anastomosis with Montgomery T-tube insertion (Fig. 1).

Preoperative vital signs were maintained within normal limits. The patient was pre-medicated with $0.2 \mathrm{mg}$ of glycopyrrolate intramuscularly $30 \mathrm{~min}$ before the induction of anesthesia. The patient was monitored using a non-invasive measurement of blood pressure, electrocardiogram, pulse oxymetry, endtidal carbon dioxide concentration, direct invasive arterial pressure, neuromuscular relaxation monitoring by train-offour ratio, and bispectral index (as measured using a BIS ${ }^{\mathrm{TM}}$ monitor 2000; Aspect Medical Systems, Norwood, MA, USA). Induction of anesthesia was carried out using midazolam (5 $\mathrm{mg})$ and rocuronium $(0.6 \mathrm{mg} / \mathrm{kg})$ intravenously. Anesthesia was maintained by the continuous infusion with propofol (0.05$0.1 \mathrm{mg} / \mathrm{kg} / \mathrm{min}$, Fresofol ${ }^{\circledR} 2 \%$; Fresenius Kabi, Austria) and remifentanil (0.1-0.5 ug/kg/min, Ultiva ${ }^{\mathrm{TM}}$; GlaxoSmithKline,

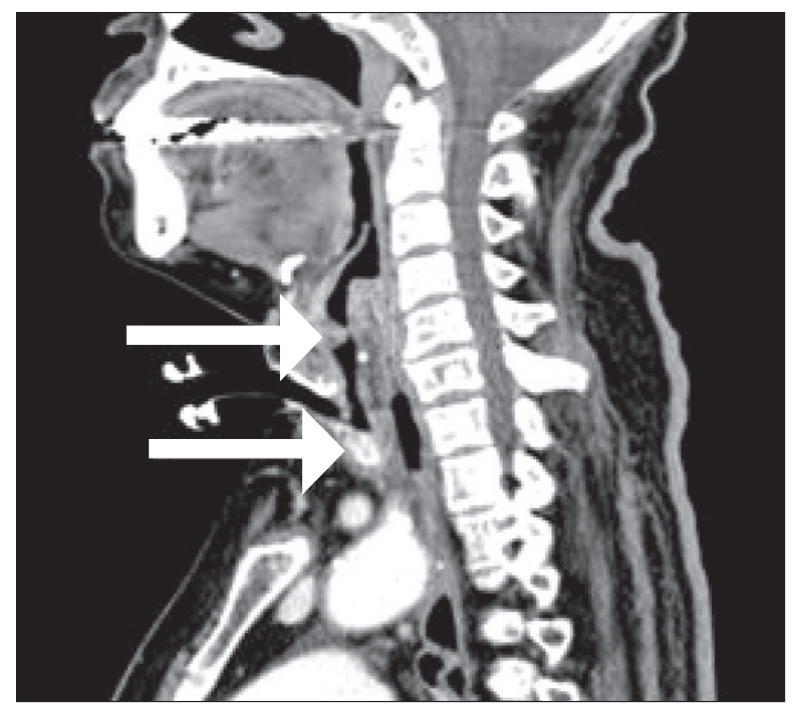

Fig. 1. Computed tomography demonstrating subglottic stenosis (upper arrow) and tracheal narrowing (lower arrow).
London, UK) with a two infusion Terufusion ${ }^{\text {TM }}$ syringe pump (Terumo, Tokyo, Japan). Fraction of inspired oxygen was $0.8-1.0$ and $\mathrm{SpO}_{2}$ was maintained at $100 \%$ during operation. During anesthesia, the infusion rate of propofol was titrated under guidance by the bispectral index score from $40-60$. The infusion rate of remifentanil was titrated with baseline vital signs.

After an anterior collar incision, the surgeon removed the exposed anterior and lateral aspects of the cricoid arch using a small rongeur. The damaged and stenotic lesion was extended for about $5 \mathrm{~cm}$ and was mobilized circumferentially. The trachea was then transected at the distal end of the lesion, and the distal airway was intubated with an aseptic endotracheal tube. The end-to-end anastomosis was accomplished using a 3-0 polydioxanone interrupted suture from the membranous portion of the trachea and a Montgomery T-tube (Silicone tracheal t-tube, $10 \mathrm{~mm}$; Sewoon Medical, Seoul, Korea) had been placed prior to the complete anastomosis of the anterior portion. The tube comprised an inner diameter of $9 \mathrm{~mm}$, short $3 \mathrm{~cm}$ laryngeal portion, long $4 \mathrm{~cm}$ tracheal portion and $4 \mathrm{~cm}$ extratracheal portion (Fig. 2 and 3). At the end of the anastomosis strap, muscle interposition was done at the lateral and posterior aspects of the anastomosis site to prevent leakage or dehiscence. During the end-to-end anastomosis, insertion and extubation of the endotracheal tube, $\mathrm{SpO}_{2}$ was frequently monitored. After the Montgomery T-tube insertion, the surgeon attempted to insert a fitted endotracheal tube with an outer diameter ranging from 4.5-6.0 $\mathrm{mm}$ through the extratracheal part of the Montgomery T-tube. Because the internal diameter of the Montgomery T-tube was too narrow,

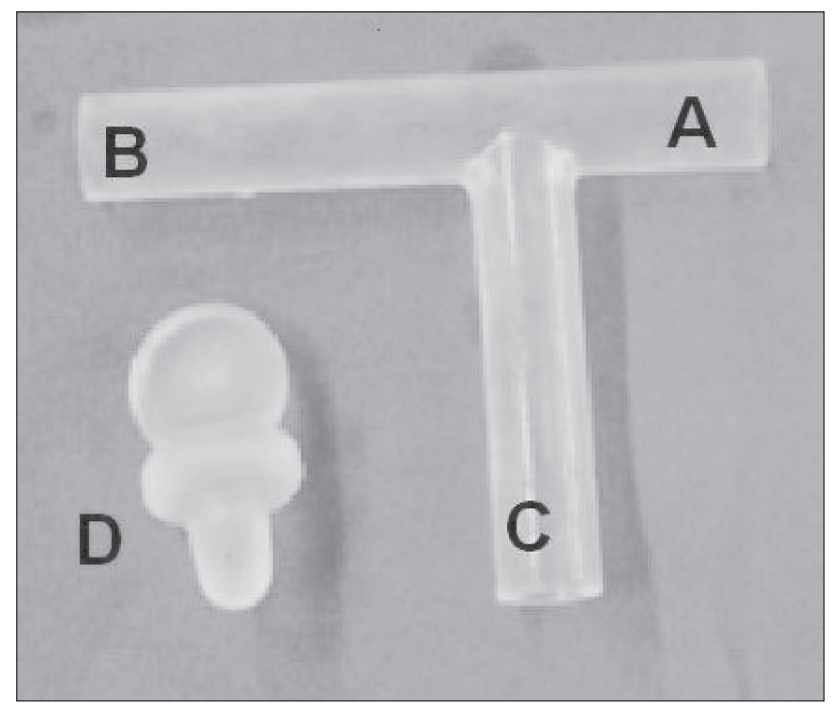

Fig. 2. The structure of a Montgomery T-tube. Symbols: A, short laryngeal portion; $\mathrm{B}$, long tracheal portion; $\mathrm{C}$, extratracheal portion; D, spigot. 


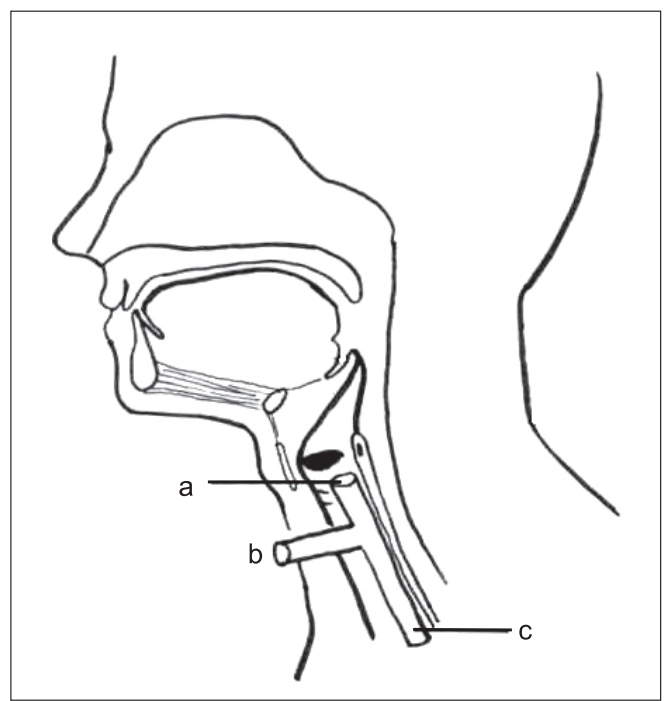

Fig. 3. Photograph of the Montgomery tube in place. Symbols: a, short laryngeal portion; $b$, extratracheal portion; c, long tracheal portion.

none of the endotracheal tubes were inserted. Therefore, the extratracheal part of Montgomery T-tube was blocked with a spigot and classic type laryngeal mask airway (LMA) with size number \#4 was inserted. The location of the Montgomery T-tube was confirmed with a bronchoscope through the LMA (Fig. 4). During the procedure, ventilation was satisfactorily maintained. At the conclusion of the procedure, the patient was ventilated spontaneously and LMA was uneventfully extubated. The patient was transferred to the intensive care unit and was managed for 1 week.

\section{Discussion}

The silicone T-tube was developed and originally described by Dr. William Montgomery in 1968 for the stenting of the airway after repair of stenosis in the low subglottic region [4]. The Montgomery T-tube is composed of a short laryngeal part, a long tracheal part, an extratracheal part and a spigot [5] (Fig. 2). The device is an uncuffed silicone T-tube that is inserted with a long limb into the trachea and a short limb protruding through the tracheostomy stoma. The tube is available in sizes ranging from 4.5-16 mm external diameter. Main indications of its use are post-laryngotracheoplasty (to keep the lumen open and to prevent mucosal laceration from scarring), tracheomalacia, relapsing polychondritis, post-tubercular bronchostenosis, amyloidosis, tracheobronchial trauma, postanastamotic bronchial stenosis and extrinsic airway compression. In contrast to a tracheotomy tube, the T-tube has advantages of preservation of the voice and normal respiration and minimal generation of cough. It avoids mucosal abrasion and the

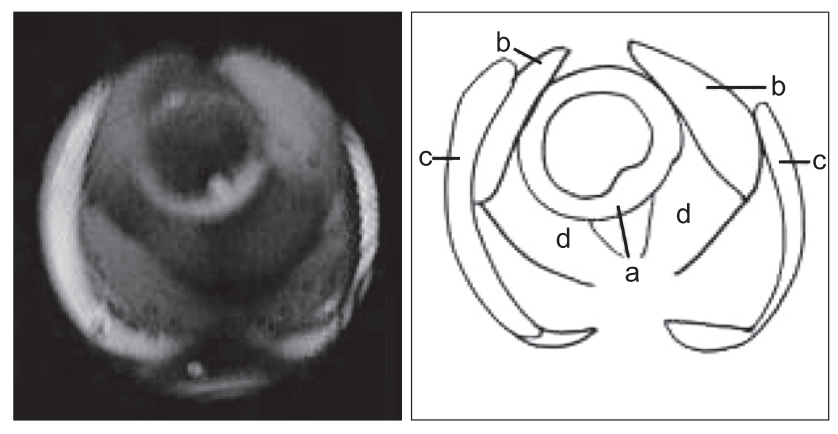

Fig. 4. Bronchoscope image of the Montgomery T-tube following LMA insertion. Symbols: a, Montgomery T-tube; b, epiglottis; c, LMA; d, vocal cord.

formation of granulation tissue associated with maintenance of a tracheotomy tube $[1,4]$. Silicone has little or no tissue reaction. It does not harden with prolonged contact with body temperature and secretion [6].

The Montgomery T-tube suffers from the disadvantage of not accommodating a standard catheter mount connector. To circumvent this, a short laryngeal portion or extratracheal segment must be occluded for ventilation. The attending anesthesiologist, therefore, should seek ways of delivering volatile agent and carrier gases [7]. After insertion of a Montgomery T-tube, airway management can be done in two ways. First, the extratracheal stem of T-tube can be blocked with a spigot, allowing the patient to maintain ventilation through the intratracheal part. Second, the airway can be maintained by inserting the endotracheal tube through the extratracheal portion. Initially we attempted the latter, but this failed because of the narrow internal diameter of the T-tube. This prompted the surgeon to insert a $10 \mathrm{~mm}$-sized Montgomery T tube because of severe subglottic stenosis. The inner diameter of the Montgomery T-tube was small, so an endotracheal tube $(6.0-4.5 \mathrm{~mm})$ was not inserted to the long tracheal part. A tube with a smaller inside diameter is able to raise airway pressure. We inserted a classic 4 LMA. The extratracheal part of the Montgomery T-tube was blocked with a spigot and ventilation was maintained. The LMA-mediated use of a fiberoptic bronchoscope can accurately localize the glottis and confirm the correct position of a Montgomery T-tube [8].

In addition to this case, there are other ways to maintain breathing after T-tube insertion. A Fogarty embolectomy catheter can be passed through the short laryngeal part to obstruct the upper intraluminal limb of the T-tube followed by insertion of the endotracheal tube into the extratracheal portion [6]. The flow of gas is then delivered through the endotracheal tube to the distal airway [1]. This procedure is complex and was not appropriate in the present case because of the absence of a connection to the extratracheal portion. Another method 
maintains ventilation via the extratracheal part of T-tube, after the occlusion of the top of the LMA [7]. Although LMA insertion is simple, this method was also presently inappropriate for the same reason. A third method is jet ventilation. In this method, the attending anesthesiologist positions the tip of a jet catheter into the short laryngeal portion to maintain ventilation. The fourth method is use of Bain's breathing system, in which a Y-connector is connected at the end of the circuit, and fresh gas flow is delivered to the extratracheal part of the T-tube and the face mask. This method carries the risk of aspiration [7]. In the present case, LMA-mediated blockage of the extratracheal part of Montgomery T-tube with a spigot as a means of maintaining ventilation was both appropriate and successful.

An anesthesiologist may not be familiar with anesthetic airway control via a Montgomery T-tube. Therefore, prior to its use, the attending anesthesiologist must understand the structure and characteristics of the Montgomery T-tube, and must have various options available for use before initiating anesthesia.

\section{References}

1. Wahidi MM, Ernst A. The Montgomery T-tube tracheal stent. Clin Chest Med 2003; 24: 437-43.

2. Kulkarni VR, Kelkar VS, Salunkhe SA. Anaesthetic challenges of the Montgomery T-tube insertion in a patient with fascioscapulohumeral dystrophy. Indian J Anaesth 2005; 49: 502-4.

3. Liu YH, Wu YC, Hsieh MJ, Ko PJ, Liu HP, Lin PJ. Montgomery T-tube insertion using a rigid bronchoscope under direct observation. ANZ J Surg 2006; 76: 853-4.

4. Cooper JD, Todd TR, Ilves R, Pearson FG. Use of the silicone tracheal T-tube for the management of complex tracheal injuries. J Thorac Cardiovasc Surg 1981; 82: 559-68.

5. Choi BR, Chung JY, Yi JW, Lee BJ, Kim DO, Kang JM. The use of the Montgomery T-tube in postprocedural subglottic stenosis repair. Korean J Anesthesiol 2009; 56: 446-8.

6. Montgomery WW. Manual for care of the Montgomery silicone tracheal T-tube. Ann Otol Rhinol Laryngol Suppl 1980; 89: 1-8.

7. Guha A, Mostafa SM, Kendall JB. The Montgomery T-tube: anaesthetic problems and solutions. Br J Anaesth 2001; 87: 787-90.

8. Tanigawa N, Sawada S, Okuda Y, Sougawa M, Komemushi A, Kojima M, et al. Expandable metallic stent placement in upper tracheal stenosis: value of laryngeal masks. AJR Am J Roentgenol 2001; 177: 1423-6. 